Tabulation of $R$ and $H$ for $\mu=(a+b \sqrt{5}) / 2$-Continued

\begin{tabular}{rrrr|rrrr|rrrr|rrrr}
$a$ & $b$ & $R$ & $H$ & $a$ & $b$ & $R$ & $H$ & $a$ & $b$ & $R$ & $H$ & $a$ & $b$ & $R$ & $H$ \\
97 & 13 & 600 & 5 & 98 & 6 & 432 & 36 & 98 & 18 & 360 & 30 & 99 & 13 & 288 & 24 \\
97 & 15 & 432 & 36 & 98 & 8 & 336 & 28 & 99 & 1 & 192 & 16 & 99 & 15 & 192 & 16 \\
97 & 17 & 288 & 24 & 98 & 10 & 336 & 28 & 99 & 3 & 192 & 16 & 99 & 17 & 240 & 20 \\
97 & 19 & 360 & 3 & 98 & 12 & 840 & 7 & 99 & 5 & 288 & 24 & 99 & 19 & 288 & 24 \\
98 & 2 & 288 & 24 & 98 & 14 & 288 & 24 & 99 & 7 & 264 & 22 & & & & \\
98 & 4 & 840 & 7 & 98 & 16 & 240 & 20 & 99 & 11 & 192 & 16 & & & &
\end{tabular}

The computation was monitored for about the first 200 tally operations to make sure the score-keeping was correct in all possible cases. The tallying was, as before, basically a question of seeing that every permutation and change in sign in the triple $\left(\xi_{1}, \xi_{2}, \xi_{3}\right)$ counted as a unit. The total running time was roughly 2.5 hours. One might remark that the human time involved in computing these class numbers $H$ from basic algebraic concepts would have to be measured in "life-times," not "man-hours."

The computation was completed 18 April 1958 and was sponsored in part by the National Science Foundation Grant G-4222.

Department of Mathematics

University of Arizona

Tucson, Arizona

1. L. E. Dickson, History of the Theory of Numbers, v. III, G. E. Stechert, New York, 1934 (for references in the first paragraph).

2. F. GöTZKY, "Über eine zahlentheoretische anwendung von modulfunktionen zweier verändlicher," Mathematische Annalen, v. 100, 1928, p. 411-437.

3. H. MAAss, "Uber die darstellung total positiver zahlen des körpers $R(\sqrt{5})$ als summe von drei quadraten," Abhandlungen aus dem Mathematischen Seminar der Hansischen Universitat, v. 14, 1941, p. 185-192.

\title{
Multiplication Time on The IBM 709
}

$$
\text { By D. D. Wall }
$$

Average multiply time is useful for roughly estimating problem running time for various problems, as well as for roughly comparing different computing machines. Determining average multiply time for the 709 is complicated, however, due to its zero-skipping feature, and requires an investigation of runs of zeros in binary sequences. The particular problem we solve is that of evaluating $R(n, l)=$ total number of runs of length $l$ in all the $2^{n}$ words of $n$ bits each, and $S(n, l)=\sum_{x=l}^{n} R(n, x)=$ number of runs of length $\geq l$ in the $2^{n}$ words of $n$ bits each. The resulting 709 average multiply time is 193 microseconds fixed point, or 170 microseconds normalized floating point, and the purpose of this note is to derive these two numbers.

We make use of a device which we call "differencing modulo 2," which obtains an $n-1$ bit number from a given $n$ bit number by writing 1 or 0 according as the successive bits in the given number exhibit a change or no change. For example, each of the (complementary) 8 bit numbers 11010001 and 00101110 gives the same result 0111001 as its 7 bit difference modulo 2 .

Received 30 April 1958. 
By differencing modulo 2, each run of length $l$ in a given word of $n$ bits gives rise to a run of 0 's of length $l-1$ in the $n-1$ bit difference, and, conversely, each $n-1$ bit number with a run of $l-10$ 's may be obtained by differencing either of two unique (complementary) $n$ bit numbers with a run of length $l$. Finally, among all the $2^{n}$ numbers of $n$ bits each, the number of runs of 0 's of length $l$ is the same as the number of runs of 1 's of length $l$, and from these considerations we conclude that

$$
R(n, l)=R(n-1, l-1)=\cdots=R(n-l+1,1),
$$

where, again, $R(n, l)=$ number of runs of length $l$ in the $2^{n}$ words of $n$ tits each.

We evaluate $R(n, 1)$ by counting the occurrences of an isolated 0 in each of the $n$ possible positions of an $n$ bit number: $2^{n-2}$ of these numbers begin $01,2^{n-3}$ of these numbers begin 101, etc., and $2^{n-2}$ of these numbers end 10 . Since the total number of runs is twice the number of runs of 0 's, we have

$R(n, 1)=2\left[2^{n-2}+(n-2) 2^{n-3}+2^{n-2}\right]=(n+2) 2^{n-2}, n>1$, and $R(1,1)=2$.

Therefore, $R(n, l)=(n-l+3) 2^{n-l-1}, n>l$, and $R(n, n)=2$.

To find the number of runs of length $\geq l$ we use the formula for summation by parts, $\sum_{a}^{b} u(x) \Delta v(x)=u(b+1) v(b+1)-u(a) v(a)-\sum_{a}^{b} v(x+1) \Delta u(x)$, and obtain

$$
S(n, l)=\sum_{x=l}^{n} R(n, x)=(n-l+2) 2^{n-l} .
$$

Now, 709 multiplication requires $6 \mu$ for each 1 bit in the multiplier plus $6 \mu$ for each run of 0 's, plus $6 \mu$ additional for each of these runs of 0 's of length 7-12, plus $6 \mu$ more for each of these runs of length $13-18$, etc., in addition to certain time charges independent of the bit structure. The average number of 1 's in an $n$ bit number, all $n$ bit numbers equally likely, is $n / 2$, and the average number of runs of 0 's is $(n+1) / 4$. This last quantity may be found by again differencing modulo 2 , since the number of runs in an $n$ bit number is 1 plus the number of 1 's in the $n-1$ bit difference.

For fixed point multiplication, $12 \mu$ are required for instruction time, $12 \mu$ for data transfer, $6 \mu$ are reserved for other purposes, and $3 \mu$ must be added as an average waiting time at the end of the operation in order to total a multiple of $12 \mu$ basic cycles. Therefore, with $n=35$ and all $n$ bit numbers assumed equally likely, average fixed point multiply time is given by $T=33+6(35 / 2)+6(36 / 4)$ $+d=192+d$, where $d=6 \cdot \frac{1}{2} \cdot 2^{-35}[S(35,7)+S(35,13)+\cdots]$ is the delay occasioned by runs of 0 's of length $>6$. On evaluating this expression we find that $d<1$ and so obtain the $193 \mu$ result. Although the 709 disposes of a zero multiplier in a different and quicker manner, this exception does not modify the $193 \mu$ answer because of the assumption that all $2^{35}$ numbers are equally likely.

For normalized floating point multiplication, the mantissa has 27 bits of which the first is a 1 , and $12 \mu$ are required to add the exponents. The resulting average time is $T=45+6(1+26 / 2)+6(27 / 4)+d=169.5+d$, and $d=6 \cdot \frac{1}{2} \cdot 2^{-26}[S(26,7)+S(26,13)+\cdots]$ is $<\frac{1}{2}$, thereby giving the $170 \mu$ result. 